INLO-PUB-2/94

\title{
Sphalerons and other saddles from cooling
}

\author{
Margarita García Pérez and Pierre van Baal \\ Instituut-Lorentz for Theoretical Physics, \\ University of Leiden, PO Box 9506, \\ NL-2300 RA Leiden, The Netherlands.
}

\begin{abstract}
We describe a new cooling algorithm for SU(2) lattice gauge theory. It has any critical point of the energy or action functional as a fixed point. In particular, any number of unstable modes may occur. We also provide insight in the convergence of the cooling algorithms. A number of solutions will be discussed, in particular the sphalerons for twisted and periodic boundary conditions which are important for the low-energy dynamics of gauge theories. For a unit cubic volume we find a sphaleron energy of resp. $\mathcal{E}_{s}=34.148(2)$ and $\mathcal{E}_{s}=72.605(2)$ for the twisted and periodic case. Remarkably, the magnetic field for the periodic sphaleron satisfies at all points $\operatorname{Tr} B_{x}^{2}=\operatorname{Tr} B_{y}^{2}=\operatorname{Tr} B_{z}^{2}$.
\end{abstract}

\section{Introduction}

Saddle points of the energy functional can play an important role in non-perturbative dynamics, as they describe the minimal barrier to be taken to go from one to the next minimum energy configuration. In a heat bath it determines the critical temperature where barrier crossing is no longer suppressed. In a strongly interacting theory it determines the coupling (or volume) where barrier crossing - quantum mechanically always allowed - is no longer exponentially suppressed. The saddle point we have in mind here is the sphaleron [1], which has precisely one unstable mode. This comes about since one determines the minimal barrier height from a mini-max procedure [2]. When one has a path in field space from one minimum to the other, one first finds the maximal energy along the path. Then one minimizes this maximum over the space of all such paths. The unstable mode will correspond to the decrease along the path that goes through this mini-max configuration. Given such a saddle point, it is often difficult to prove in all rigour that it is the one with lowest energy, as there may be local minima when varying over the space of paths.

Quite often a sphaleron lies on a particular instanton path (i.e. the action associated to the path is also minimal), but this is by no means guaranteed. When the instanton has non-trivial parameters (moduli), mini-max with respect to those parameters provides a candidate sphaleron. For $S^{3}$ one can analytically verify that this candidate is a saddle point of the energy functional with one unstable mode [3]. For $T^{3}$ this was studied numerically [4, 5. It was not entirely conclusive from that study if the sphaleron was indeed associated 
to the top of an instanton path. With the new algorithm we have been able to address this issue more directly and conclude that it is the case for both periodic and twisted [6] boundary conditions.

We will only consider the case of pure $\mathrm{SU}(2)$ gauge theories. In an infinite volume the mini-max procedure is easily seen to lead to zero sphaleron energy, as instantons can have an arbitrarily large scale parameter. In a finite volume, the scale parameter can not be larger than the volume, which stabilizes the mini-max procedure at a non-zero energy. In the presence of a Higgs field, like in the standard model [1], the scale is set by the Higgs mass.

Before discussing the numerical results we will first describe the algorithm for finding saddle points and discuss the convergence. This study is specific for $\mathrm{SU}(2)$, but the principle of taking the square of the equations of motion as the action to be minimized will work equally well for $\mathrm{SU}(\mathrm{N})$ gauge theories, with or without scalar fields. In section 4 we will discuss results for the class of analytically known solutions with constant field strength, to test the various aspects of the method. In section 5 the results for the sphalerons are presented in detail. We end the paper with some concluding remarks. Readers only interested in the results should directly go to section 5, perhaps after section 2 .

\section{The algorithm}

Consider the square of the equations of motion summed over its variables as a functional. Its absolute minima are by construction solutions of the equations of motion. Stability is now obvious, but also follows from the fact that at such a solution the Hessian of the new functional is (twice) the square of the Hessian of the energy (or action) functional. In the $n$-dimensional continuum we have the following expressions for the two functionals

$$
\begin{gathered}
S=-\frac{1}{2} \int d_{n} x \operatorname{Tr}\left(F_{\mu \nu}^{2}(x)\right), \\
\hat{S}=-2 \int d_{n} x \operatorname{Tr}\left(\left(\mathcal{D}_{\mu} F_{\mu \nu}(x)\right)^{2}\right),
\end{gathered}
$$

with $F_{\mu \nu}(x)=\partial_{\mu} A_{\nu}(x)-\partial_{\nu} A_{\mu}(x)+\left[A_{\mu}(x), A_{\nu}(x)\right]$ the Yang-Mills field strength in terms of the anti-hermitian vector potential $A_{\mu}(x)$ and $\mathcal{D}_{\mu}=\partial_{\mu}+\operatorname{ad} A_{\mu}(x)$ the covariant derivative.

This was considered in the past for constructing monopole solutions in three dimensions [7], but the process of finding the minimum of $\hat{S}$ was not gauge invariant. Like for the Wilson action [8]

$$
S=N^{4-n} \sum_{x, \mu, \nu} \operatorname{Tr}\left(1-\underset{x^{\nu}}{\nu}\right)=N^{4-n} \sum_{x, \mu, \nu} \operatorname{Tr}\left(1-U_{\mu}(x) U_{\nu}(x+\hat{\mu}) U_{\mu}^{\dagger}(x+\hat{\nu}) U_{\nu}^{\dagger}(x)\right)
$$

a gauge invariant lattice action for the square of the equations of motion is not too difficult to write down 9

$$
\hat{S}=N^{6-n} \sum_{x, \mu} \operatorname{Re} \operatorname{Tr}\left(\tilde{U}_{\mu}(x) \tilde{U}_{\mu}^{\dagger}(x)-\left(U_{\mu}(x) \tilde{U}_{\mu}^{\dagger}(x)\right)^{2}\right)
$$

where $U_{\mu}(x)$ is a group element on the link that runs from $x$ to $x+\hat{\mu}(\hat{\mu}$ is the unit vector in the $\mu$ direction), $N$ is the number of lattice points in one of the directions and $\tilde{U}_{\mu}(x)$ is 
the sum over $2(n-1)$ staples

$\tilde{U}_{\mu}(x)=\sum_{\nu \neq \mu}\left(\nu_{x}^{\mu}+\stackrel{\nu}{\nu}_{\mu}^{x_{\mu}}\right)=\sum_{\nu \neq \mu}\left(U_{\nu}(x) U_{\mu}(x+\hat{\nu}) U_{\nu}^{\dagger}(x+\hat{\mu})+U_{\nu}^{\dagger}(x-\hat{\nu}) U_{\mu}(x-\hat{\nu}) U_{\nu}(x+\hat{\mu}-\hat{\nu})\right)$,

To derive the lattice equations of motion, we observe that $S$ depends on $U_{\mu}(x)$ through the expression:

$$
S\left(U_{\mu}(x)\right)=2 N^{4-n} \operatorname{Re} \operatorname{Tr}\left(1-U_{\mu}(x) \tilde{U}_{\mu}^{\dagger}(x)\right) .
$$

As $\tilde{U}_{\mu}(x)$ is independent of $U_{\mu}(x)$, it follows that

$$
U_{\mu}(x) \tilde{U}_{\mu}^{\dagger}(x)-\tilde{U}_{\mu}(x) U_{\mu}^{\dagger}(x)=0
$$

Its square, summed over the lattice, yields $\hat{S}$. For SU(2) one can also use the fact that the equations of motion are solved by

$$
U_{\mu}(x)= \pm \tilde{U}_{\mu}(x) /\left\|\tilde{U}_{\mu}(x)\right\| \quad, \quad\left\|\tilde{U}_{\mu}(x)\right\|^{2} \equiv \frac{1}{2} \operatorname{Tr}\left(\tilde{U}_{\mu}(x) \tilde{U}_{\mu}^{\dagger}(x)\right)
$$

where only the positive sign will allow for solutions that have a smooth continuum limit. We can now introduce an error functional [5]

$$
\tilde{S}=N^{6-n} \sum_{x, \mu} \operatorname{Tr}\left(1-U_{\mu}(x) \tilde{U}_{\mu}^{\dagger}(x) /\left\|\tilde{U}_{\mu}(x)\right\|\right)
$$

In the continuum limit one finds $\tilde{S}=\frac{1}{16}(n-1)^{-2} \hat{S}$. This follows in the usual way [4] from expanding the links in $a=1 / N$, defining the relation between the vector potential in the continuum and the link variables on the lattice by

$$
U_{\mu}(x)=\operatorname{Pexp}\left(\int_{0}^{a} A_{\mu}(x+s \hat{\mu}) d s\right) .
$$

One finds after some algebra

$$
U_{\mu}(x) \tilde{U}_{\mu}^{\dagger}(x) /\left\|\tilde{U}_{\mu}(x)\right\|=\exp \left(-\frac{1}{2(n-1) N^{3}} \sum_{\nu} \mathcal{D}_{\nu} F_{\mu \nu}(x)+\mathcal{O}\left(N^{-5}\right)\right) .
$$

We can instead of the standard Wilson action use any improved action, containing $n \times m$ Wilson loops with appropriate couplings, such as the over-improved actions we have used in ref. 4

$$
S(\varepsilon)=\frac{4-\varepsilon}{3} \sum_{x, \mu, \nu} \operatorname{Tr}(1-\nu \sqrt{x})+\frac{\varepsilon-1}{48} \sum_{x, \mu, \nu} \operatorname{Tr}(1-\nu
$$

All that needs to be modified in the above is the definition of $\tilde{U}_{\mu}(x)$, which will now also contain the staples associated with the larger Wilson loops appearing in eq. (12)

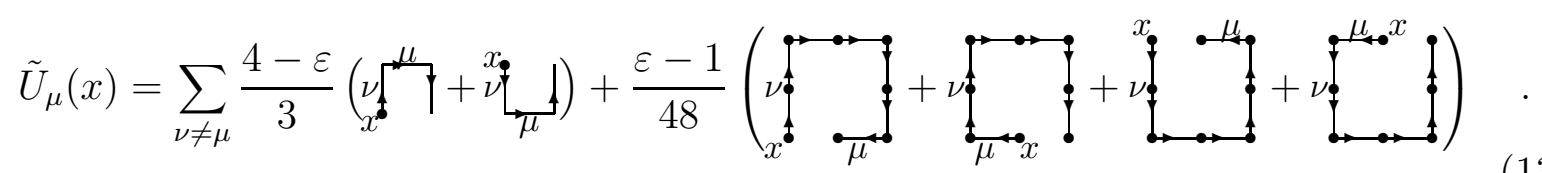


Like for $S(\varepsilon)$, the $\varepsilon$ dependence drops out in the continuum limit for $\hat{S}(\varepsilon)$. However, for $\tilde{S}$ one finds in this limit $\tilde{S}(\varepsilon)=36((n-1)(31-7 \varepsilon))^{-2} \hat{S}$.

The ordinary cooling algorithm [10] for both of these actions is based on the observation that the replacement

$$
U_{\mu}^{\prime}(x)=\tilde{U}_{\mu}(x) /\left\|\tilde{U}_{\mu}(x)\right\|
$$

minimizes $S\left(U_{\mu}(x)\right.$ ). Note that for $S$ (resp. $S(\varepsilon)$ ) cooling we should require the lattice to be at least two (resp. three) sites in each direction. To completely specify the algorithm one also should prescribe in what order every link is being updated. One sweep is the process of updating each link precisely once in that particular order. This defines a mapping $\left(U \equiv\left\{U_{\mu}(x)\right\}\right)$

$$
U^{\prime}=T(U) \quad, \quad T=T_{\mathcal{N}} \circ T_{\mathcal{N}-1} \circ \cdots \circ T_{2} \circ T_{1},
$$

where $T_{i}$ is the single-link update of eq. (14) and the label $i$ stands for the order in which the $\mathcal{N}=n N^{n}$ different links are being updated. It is actually not difficult to compute exactly by which amount the action is decreasing. For the single-link update we find

$$
S\left(U_{\mu}^{\prime}(x)\right)-S\left(U_{\mu}(x)\right)=-2 N^{4-n}\left\|\tilde{U}_{\mu}(x)\right\|\left\|U_{\mu}^{\prime}(x)-U_{\mu}(x)\right\|^{2},
$$

where we made use of eq. (14). To find the change of the action after one sweep, one has to simply add the contributions of each single-link update keeping in mind, however, that the value of $\left\|\tilde{U}_{\mu}(x)\right\|$ depends on which links had been updated before. In the continuum limit $\left\|\tilde{U}_{\mu}(x)\right\| \rightarrow 2(n-1)$ and to a good accuracy the action changes from one sweep to the next by the amount

$$
S(T(U))-S(U) \sim-4(n-1) N^{4-n}\|T(U)-U\|^{2} \equiv-4(n-1) N^{4-n} \sum_{x, \mu}\left\|U_{\mu}^{\prime}(x)-U_{\mu}(x)\right\|^{2} .
$$

For saddle points, which are fixed points of this algorithm, one can of course lower the action further and the algorithm is necessarily unstable.

For the functional $\hat{S}$ any solution corresponds to the absolute minimum $\hat{S}=0$, and cooling with this functional should hence not lead to any instability for the saddle points of the original action, eqs. (33,12). Unfortunately, it can be proven that one cannot analytically minimize $\hat{S}$ as a function of one of its links. All that is required, however, is that the functional is lowered if and only if we are not at a fixed point. We will see, as our intuition tells us, that this is a sufficient condition for convergence of the algorithm (necessity should be obvious). Let us nevertheless write down the necessary ingredients to minimize the single-link dependence of $\hat{S}$, which was already considered by Van der Sijs [9]. We restrict ourselves in this paper to the version of $\hat{S}$ derived from the Wilson action, as the analysis is prohibitively more complicated for improved actions (for $\tilde{S}$ the situation is in either case intractable, because of the appearance of $\left\|\tilde{U}_{\mu}(x)\right\|$ in the denominator of eq.(可)). The analogue of eq. (6) becomes

$$
\hat{S}\left(U_{\mu}(x)\right)=N^{6-n} \operatorname{Re} \operatorname{Tr}\left(U_{\mu}^{\dagger}(x) W_{\mu}(x)-3 \sum_{\alpha=1}^{2(n-1)}\left(U_{\mu}^{\dagger}(x) V_{\mu}^{\alpha}(x)\right)^{2}-\left(U_{\mu}^{\dagger}(x) \tilde{U}_{\mu}(x)\right)^{2}\right),
$$

where the index $\alpha$ runs over the $2(n-1)$ different staples of eq. (5), such that $\tilde{U}_{\mu}(x)=$ $\sum_{\alpha} V_{\mu}^{\alpha}(x)$. The expression for $W_{\mu}(x)$ is a complicated combination of planar and non- 
planar Wilson loops, which are best exhibited in terms of unit vectors $\hat{a}, \hat{b} \in\{ \pm \hat{1}, \cdots, \pm \hat{n}\}$

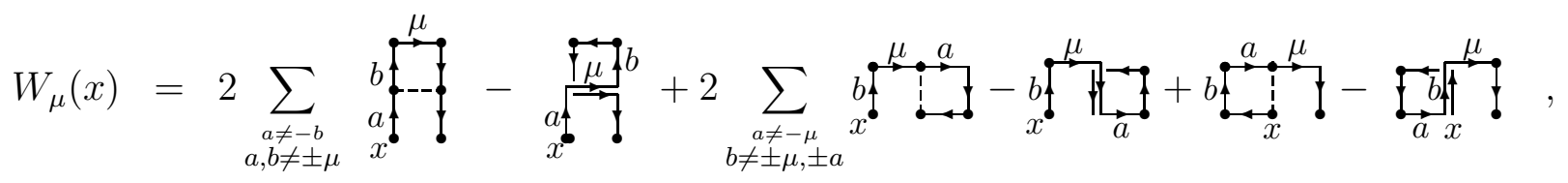

or in explicit form, using the convention that $U_{a}(x) \equiv U_{-a}^{\dagger}(x-\hat{a})$ :

$$
\begin{aligned}
W_{\mu}(x) & =2 \sum_{a, b \neq \pm \mu ; a \neq-b}\left\{U_{a}(x) U_{b}(x+\hat{a}) U_{\mu}(x+\hat{a}+\hat{b}) U_{b}^{\dagger}(x+\hat{a}+\hat{\mu}) U_{a}^{\dagger}(x+\hat{\mu})\right. \\
& \left.-U_{a}(x) U_{\mu}(x+\hat{a}) U_{b}(x+\hat{a}+\hat{\mu}) U_{\mu}^{\dagger}(x+\hat{a}+\hat{b}) U_{b}^{\dagger}(x+\hat{a}) U_{\mu}(x+\hat{a}) U_{a}^{\dagger}(x+\hat{\mu})\right\} \\
& +2 \sum_{b \neq \pm \mu, \pm a ; a \neq-\mu}\left\{U_{b}(x) U_{\mu}(x+\hat{b}) U_{a}(x+\hat{b}+\hat{\mu}) U_{b}^{\dagger}(x+\hat{a}+\hat{\mu}) U_{a}^{\dagger}(x+\hat{\mu})\right. \\
& -U_{b}(x) U_{\mu}(x+\hat{b}) U_{b}^{\dagger}(x+\hat{\mu}) U_{a}(x+\hat{\mu}) U_{b}(x+\hat{a}+\hat{\mu}) U_{a}^{\dagger}(x+\hat{b}+\hat{\mu}) U_{b}^{\dagger}(x+\hat{\mu}) \\
& +U_{a}^{\dagger}(x-\hat{a}) U_{b}(x-\hat{a}) U_{a}(x+\hat{b}-\hat{a}) U_{\mu}(x+\hat{b}) U_{b}^{\dagger}(x+\hat{\mu}) \\
& \left.-U_{b}(x) U_{a}^{\dagger}(x+\hat{b}-\hat{a}) U_{b}^{\dagger}(x-\hat{a}) U_{a}(x-\hat{a}) U_{b}(x) U_{\mu}(x+\hat{b}) U_{b}^{\dagger}(x+\hat{\mu})\right\}
\end{aligned}
$$

The sum in this expression is restricted to those combinations of $\hat{a}$ and $\hat{b}$ that do not lead to any backtracking in the Wilson loop defined by the products of the links. If one tries to minimize $\hat{S}\left(U_{\mu}(x)\right)$ with respect to $U_{\mu}(x)$ under the constraint $\left\|U_{\mu}(x)\right\|=1$, one finds

$$
\lambda U_{\mu}(x)=M\left(U_{\mu}(x)\right)-W_{\mu}(x)
$$

Note that the lattice should now be at least three sites in each direction. The operator $M$, whose $\mu$ and $x$ dependence was suppressed, is defined for $\mathrm{SU}(2)$ through the equation

$$
M\left(U_{\mu}(x)\right) \equiv 6 \sum_{\alpha} \operatorname{Tr}\left(U_{\mu}(x) V_{\mu}^{\alpha}(x)^{\dagger}\right) V_{\mu}^{\alpha}(x)+2 \operatorname{Tr}\left(U_{\mu}(x) \tilde{U}_{\mu}^{\dagger}(x)\right) \tilde{U}_{\mu}(x)
$$

An attempt to solve for $\lambda$, using the condition that $U_{\mu}(x)$ is unitary, leads to a complicated eighth order polynomial, and solving it numerically for each update would be too costly. Instead, we define our update by the simple equation

$$
U_{\mu}^{\prime}(x)=\frac{M\left(U_{\mu}(x)\right)-W_{\mu}(x)}{\left\|M\left(U_{\mu}(x)\right)-W_{\mu}(x)\right\|},
$$

The new feature is that the r.h.s. still depends on $U_{\mu}(x)$ and that we do not obtain a minimum of eq. (18). Nevertheless, this is harmless since some algebra will show that our update does lower $\hat{S}$ and as we argued above, and shall show in some more detail in the next section, this is sufficient. One finds

$$
\delta \hat{S}\left(U_{\mu}(x)\right)=-N^{6-n}\left(\left\|M\left(U_{\mu}(x)\right)-W_{\mu}(x)\right\|\left\|\delta U_{\mu}(x)\right\|^{2}+<\delta U_{\mu}(x), M\left(\delta U_{\mu}(x)\right)>\right),
$$

where, of course, $\delta U_{\mu}(x) \equiv U_{\mu}^{\prime}(x)-U_{\mu}(x), \delta \hat{S}\left(U_{\mu}(x)\right) \equiv \hat{S}\left(U_{\mu}^{\prime}(x)\right)-\hat{S}\left(U_{\mu}(x)\right)$ and

$$
<\delta U_{\mu}(x), M\left(\delta U_{\mu}(x)\right)>=3 \sum_{\alpha}\left|\operatorname{Tr}\left(\delta U_{\mu}^{\dagger}(x) V_{\mu}^{\alpha}(x)\right)\right|^{2}+\left|\operatorname{Tr}\left(\delta U_{\mu}^{\dagger}(x) \tilde{U}_{\mu}(x)\right)\right|^{2}
$$

In the continuum limit one has $\left\|M\left(U_{\mu}(x)\right)-W_{\mu}(x)\right\| \rightarrow 8(n-1)(2 n+1)$. 
We note that lattice artefact solutions for $\hat{S}$ will not be eliminated by eq. (22). For example, putting one link to minus the identity (while keeping all others equal to the identity) is a fixed point of eq. (22). It is, however, not a fixed point for eq. (14). In practise we first use $S$ cooling to bring the energy (or action) down to values not too much above where we expect a saddle point to occur, after which the $\hat{S}$ cooling will in general bring it to a smooth solution. One can also combine the $S$ and $\hat{S}$ cooling, by replacing $U_{\mu}(x)$ on the r.h.s. of eq. (22) with $\tilde{U}_{\mu}(x) /\left\|\tilde{U}_{\mu}(x)\right\|$. We have not proven that this always lowers $\hat{S}$ (although it did in all cases we considered).

\section{Convergence}

We will study the convergence of the algorithm by considering the linear approximation for the mapping $T$ in eq. (15). For $S$ (or $S(\varepsilon)$ ) cooling, $T_{i}$ will correspond to eq. (14), while for $\hat{S}$ cooling it will correspond to eq. (22). To warm up we first consider the singlelink convergence of the latter, while keeping all links (except $U_{\mu}(x)$ ) fixed. For notational convenience we drop the $\mu$ and $x$ dependence. Let us assume that $U_{o}$ is a fixed point, and $X$ is a small element of the $\mathrm{SU}(2)$ Lie-algebra. We now consider $U=e^{X} U_{o}$ and compute $X^{\prime}$ to linear order in $X$ from $U^{\prime} \equiv e^{X^{\prime}} U_{o}$. After some algebra one finds

$$
X^{\prime}=\left\|M\left(U_{o}\right)-W\right\|^{-1}\left(6 \sum_{\alpha} \operatorname{Tr}\left(X Y_{\alpha}^{\dagger}\right) Y_{\alpha}+2 \operatorname{Tr}\left(X \tilde{Y}^{\dagger}\right) \tilde{Y}\right),
$$

where $\tilde{Y} \equiv \sum_{\alpha} Y_{\alpha}$ and $Y_{\alpha}=\operatorname{Im}\left(U_{o}^{\dagger} V_{\alpha}\right) \equiv \frac{1}{2 i}\left(U_{o}^{\dagger} V_{\alpha}-V_{\alpha}^{\dagger} U_{o}\right)$. In the continuum limit this is easily seen to imply that $\left\|X^{\prime}\right\| /\|X\|=\mathcal{O}\left(N^{-4}\right)$ and we have verified that the single-link iteration is indeed extremely efficient. One iteration typically brings $U$ already very close to the minimum of eq. (18) (although we would like to emphasize again that this is not really important, as the other links that contribute to $\hat{S}$ will be changed during the sweep).

With respect to the $\mathrm{SU}(2)$ Lie-algebra basis $i \tau_{k} / 2\left(\tau_{k}\right.$ the Pauli matrices) eq. (25) is symmetric, but this is in general no longer true for the linear approximation of $T$, which we will denote by $d T$. The latter is determined exactly as above by evaluating $U^{\prime}=T\left(e^{X} U_{o}\right)$ and extracting $X_{\mu}^{\prime}(x)$ to linear order in $X$ from $\exp \left(X_{\mu}^{\prime}(x)\right)=U_{\mu}^{\prime}(x) U_{\mu}^{o}(x)^{\dagger}$, where $U_{o}$ is the fixed point. Numerically this is easily achieved by first obtaining the fixed point to a high accuracy, assuming the last value of $U$ can be equated to $U_{o}$, and then looking at the response of one complete sweep to $U=e^{X} U_{o}$, if necessary doing extrapolation in $X \rightarrow 0$ to extract the part linear in $X$. One might be tempted to prove that $T$ is a socalled contraction, for which it is required that $\|T(V)-T(U)\| \leq \kappa\|V-U\|$, with $\kappa<1$. The contraction theorem asserts that in such a case there is a unique fixed point of the mapping $T$. At the linear level, near the fixed point, this would imply that $d T^{t} d T$ (which is symmetric and positive definite) has no eigenvalues bigger or equal to one. We know, however, that the fixed point is neither globally nor locally unique. Indeed, for all cases we studied there are plenty of eigenvalues bigger than one. If $d T$ would have been symmetric, there would have also been eigenvalues of $d T$ that are bigger (in absolute value) than one, which would clearly be in contradiction with the fact that the action is lowered with each update.

As $d T$ is not a symmetric matrix, we can not diagonalize it. Still, it can over the complex numbers be brought to the so-called Jordan-normal form by a non-singular (complex) 
transformation $A$

$$
d T=A^{-1} J A \equiv A^{-1}\left(\begin{array}{ccc}
J_{1} & & \ominus \\
& \ddots & \\
\ominus & & J_{\ell}
\end{array}\right) A
$$

where $J_{i}$ is the smallest Jordan block, of the form

$$
J_{i} \equiv\left(\begin{array}{cccc}
\lambda_{i} & 1 & & \ominus \\
& \ddots & \ddots & \\
& & \ddots & 1 \\
\ominus & & & \lambda_{i}
\end{array}\right)
$$

with $\lambda_{i}$ the eigenvalues. When complex, they occur in conjugate blocks. Different blocks in our conventions may have the same $\lambda_{i}$, and blocks of dimension 1 correspond to eigenvalues with equal left and right eigenvectors. As $d T^{k}=A^{-1} J^{k} A$, any block with $\left|\lambda_{i}\right|<1$ will approach zero. Blocks with $\left|\lambda_{i}\right|>1$ would grow without bound and can never be consistent with the fact $\hat{S}$ is a decreasing functional as a function of the sweeps. What remains are the eigenvalues $\left|\lambda_{i}\right|=1$. They can, and will, occur for the following reason. Suppose $X$ is a zero mode for the Hessian of $\hat{S}$ at the fixed point $U_{o}$, then $\hat{S}\left(e^{X} U_{o}\right)-\hat{S}\left(U_{o}\right)=\mathcal{O}\left(X^{4}\right)$. On the other hand using eq. (23), $\hat{S}\left(e^{X^{\prime}} U_{o}\right)-\hat{S}\left(e^{X} U_{o}\right)=\mathcal{O}\left(\left\|X^{\prime}-X\right\|^{2}\right)$, which can only be consistent if $X^{\prime}=X$. Consequently $X$ is an eigenvector of $d T$ with eigenvalue 1 . The converse is also true, i.e. an eigenvector with eigenvalue 1 is a zero-mode of the Hessian. Consequently, there are at least as many unit eigenvalues as there are gauge degrees of freedom. To be precise, there are $3 N^{n}$ minus the dimension of $\operatorname{Stab}\left(U_{0}\right)$ unit eigenvalues. ( $\operatorname{Stab}\left(U_{0}\right)$ is the stabilizer of $U_{o}$, which is the subgroup of the gauge group that leaves $U_{o}$ unchanged. It will only for very special configurations be non-trivial). It is slightly more subtle to argue that $\left|\lambda_{i}\right|=1$ is only compatible with eq. (22) if $\lambda_{i}=1$ (one uses the fact that there can be no linear relation among the eigenvectors, because $A$ is non-singular, which implies that after each sweep the amount by which $\hat{S}$ is lowered is bounded away from zero if $\lambda_{i} \neq 1$ ). It is more easy to rule out that $\lambda_{i}=1$ can occur in blocks $J_{i}$ of dimension greater than 1 . In other words, left and right eigenvectors for the eigenvalue equal to 1 always will coincide.

Clearly, the fixed point is only unique up to the gauge degrees of freedom and the moduli of the fixed point (by moduli we mean the gauge invariant parameter space along which $\hat{S}=0$ and consequently along which $S$ is degenerate), and there is a one to one relation with the $\lambda_{i}=1$. These do not influence the convergence of the algorithm. But it is important to realize that one has no control over which fixed point will be obtained. (The gauge in which the fixed point is obtained will entirely be determined by the starting configuration, as $T$ commutes with applying a gauge transformation, i.e. $T\left({ }^{g} U\right)={ }^{g} T(U)$.) It can happen that there are zero-modes of the Hessian that are not associated to a moduli parameter of the fixed point, i.e. $\hat{S}$ is no longer degenerate to fourth (or higher) order. In that case one needs to go beyond the linear approximation of $T$ and convergence is worse than exponential. In the generic case, however, the rate in which $\hat{S}$ decreases is determined by the gap $\hat{\mu}$ in $\left|\lambda_{i}-1\right|$, or after $k$ sweeps $\hat{S}$ decreases the next sweep by an amount $\delta \hat{S} \sim \exp (-2 k \hat{\mu})$. The same argument holds of course for $S$ cooling to a (local) 
minimum, replacing the gap $\hat{\mu}$ by its appropriate value $(\mu)$ obtained from the mapping $T$ for $S$ cooling.

We can find a relation between the values of these gaps and the minimal non-trivial curvature of $S$ at the fixed point if we assume that the gap is due to a real eigenvalue and its eigenvector coincides with the fluctuation along the direction of minimal curvature. The associated zero-point frequency will be denoted by $\omega$ (scaled so as to have the appropriate continuum limit). At a fixed point $U_{o}$ one has for $X_{\mu}(x) \equiv i \delta A_{\mu}^{a}(x) \tau_{a} /(2 N)$

$$
S\left(e^{X} U_{o}\right)=S\left(U_{o}\right)+\frac{1}{2} N^{-n} \sum_{x \mu a ; y \nu b} \delta A_{\mu}^{a}(x) H_{\mu \nu}^{a b}(x, y) \delta A_{\nu}^{b}(y)+\mathcal{O}\left(\delta A^{3}\right)
$$

which provides the definition for the Hessian $H_{\mu \nu}^{a b}(x, y)$ as a $3 n N^{n}$ square matrix, whose eigenvalues will scale to the appropriate continuum limit for $N \rightarrow \infty$. Hence, $\omega^{2}$ equals the lowest non-zero eigenvalue $\left(\lambda_{m}\right)$ of the Hessian. It can be easily shown that for any saddle point of $S$, such that $\hat{S}\left(U_{o}\right)=0$, the functional $\hat{S}$ has the expansion

$$
\hat{S}\left(e^{X} U_{o}\right)=\frac{1}{2} N^{-n} \sum_{x \mu a ; y \nu b} \delta A_{\mu}^{a}(x) \hat{H}_{\mu \nu}^{a b}(x, y) \delta A_{\nu}^{b}(y)+\mathcal{O}\left(\delta A^{3}\right), \quad \hat{H}=2 H^{2},
$$

where by $H^{2}$ we mean of course the matrix multiplication of the Hessian of $\mathrm{S}$ with itself. In the same shorthand notation (i.e. dropping the indices), we assume that under the $S$ cooling $H \cdot X=\lambda_{m} X$ and $X^{\prime} \equiv d T \cdot X=(1-\mu) X$, which allows us to calculate the change in the action both from eq. (28), giving $\delta S=-4 \omega^{2} \mu\left(1-\frac{1}{2} \mu\right) N^{2-n}\|X\|^{2}$ and from eq. (17), giving $\delta S=-4(n-1) \mu^{2} N^{4-n}\|X\|^{2}$. Equating these results gives

$$
\mu \sim \frac{\omega^{2}}{(n-1) N^{2}} \equiv \frac{\lambda_{m}}{(n-1) N^{2}}
$$

Similarly, using eqs. 23,29) one finds for cooling with the functional $\hat{S}$

$$
\hat{\mu} \sim \frac{\omega^{4}}{(n-1)(2 n+1) N^{4}} \equiv \frac{\lambda_{m}^{2}}{(n-1)(2 n+1) N^{4}} \quad .
$$

This is of course in accordance with ones intuition that the algorithm can slow down considerably if the functional has directions in which it is very shallow. Note that our results indicate that slowing down of the algorithm goes proportional to $N^{2+n}$ for $S$ cooling and proportional to $N^{4+n}$ for $\hat{S}$ cooling (in both cases a factor $N^{n}$ comes from the number of links to be updated in a single sweep).

Finally, most of the moduli for a continuum solution are removed by lattice artefacts, although we will discuss in the next section that in some cases a few of them can survive on a lattice. An important example of moduli that are removed by lattice artefacts are those that are related to the translational invariance of the continuum theory on a torus. As this breaking is due to the fact that integrals in the continuum are replaced by sums on the lattice, one can easily argue, as in ref. [1], that the breaking is very mild and goes as $\exp (-\rho N)$ where $\rho$ is the typical scale of the continuum solution in units of the length $L$ of the torus. For small lattices these translational modes can cause some trouble, but those lattices are usually anyhow too small to extract reliable information about the continuum limit. In ref. [4 we made judicious use of the dependence of the lattice action on the 
moduli parameters, by using the over-improved action $S(\varepsilon=-1)$ (see eq. (12)) so as to ensure that the instanton with the maximal scale parameter allowed by the finite volume is obtained as a local minimum for the action. With $S$ cooling we could then find rather accurate lattice solutions, whose scaling to a continuum solution was studied in detail. One of the advantages of the $\hat{S}$ cooling is that one can verify if the fixed point has been reached, as $\hat{S}$ should approach zero. This can, of course, also be used as a check under $S$ cooling. For example, we performed for an instanton on an $8^{3} \times 24$ lattice cooling for about 6600 sweeps, which allowed for a very precise exponential fit to the decay of the action. The effective value for $\mu$ deduced from this fit is $9.8 \times 10^{-5}$ (see fig. 3 of ref. (4⿴囗十)). We find for the best configuration of this four dimensional instanton (see table I of ref. [4]) $\hat{S}(\varepsilon=-1)=4.8 \times 10^{-4}$ and for $\tilde{S}(\varepsilon=-1)=1.3 \times 10^{-6}$. The results obtained with $S(\varepsilon=0)$ cooling on this configuration (see the same table), lead to $\hat{S}(\varepsilon=0)=2.0 \times 10^{-6}$, $\tilde{S}(\varepsilon=0)=8.2 \times 10^{-9}$, and effectively $\mu \sim 10^{-6}$ (which can be understood since for $S(\varepsilon=0$ ) the $\mathcal{O}\left(a^{2}\right)$ corrections to the action are canceled, removing to this order the dependence of the action on the scale parameter. Using the heuristic estimates of $\mu$ discussed above we find $\mu \sim \omega^{2} /\left(3 N^{2}\right)$. Since $\omega(\varepsilon \neq 0)=\mathcal{O}\left(N^{-1}\right)$, whereas $\omega(\varepsilon=0)=\mathcal{O}\left(N^{-2}\right)$, one obtains with $N=8$ the right orders of magnitude for $\mu$ ). In the remainder we will discuss the new results for $n=3$, i.e. results for the Yang-Mills energy functional.

\section{Results: Constant solutions}

We will first describe the case of constant curvature solutions, which are known analytically both on the lattice and in the continuum. We take from ref. [11] the following results. If we introduce the so-called twist tensor $n_{i j}$, which is antisymmetric and takes integer values (equivalently specified by the magnetic flux $m_{i}=\frac{1}{2} \varepsilon_{i j k} n_{j k}$ ), the following configuration is a solution on a symmetric three torus of length $L=1$, or a symmetric lattice with $N^{3}$ sites

$$
A_{j}^{o}(x)=i\left(-\pi n_{j k} x_{k}+C_{j}\right) \frac{\tau_{3}}{2} \quad, \quad U_{i}^{o}(x)=\exp \left(A_{i}^{o}(x / N) / N\right)
$$

These fields are periodic up to a gauge transformation, to be specific [12]

$$
A_{j}(x+\hat{k})=\Omega_{k}(x)\left(A_{j}(x)+\partial_{j}\right) \Omega_{k}^{-1}(x) \quad, \quad U_{j}(x+N \hat{k})=\Omega_{k}(x / N) U_{j}(x) \Omega_{k}^{-1}((x+\hat{j}) / N),
$$

where

$$
\Omega_{k}(x)=\exp \left(\frac{1}{2} i \pi x_{j} n_{j k} \tau_{3}\right) .
$$

If some of the components of the twist matrix are odd, quantities that transform in the fundamental representation of SU(2) are anti-periodic, which can be most easily seen from the Polyakov line

$$
P_{j}(x)=\frac{1}{2} \operatorname{Tr}\left\{\mathrm{P} \exp \left(\int_{0}^{1} d s A_{j}^{o}(x+s \hat{j})\right) \Omega_{j}(x)\right\}=\cos \left(\frac{1}{2} C_{j}-\pi n_{j k} x_{k}\right) .
$$

The identical result follows on the lattice, after replacing $x$ by $x / N$. For the continuum Yang-Mills and lattice Wilson actions (energies) we find

$$
S=\pi^{2} \sum_{i j} n_{i j}^{2} \quad, \quad S=4 N^{4} \sum_{i j} \sin ^{2}\left(\frac{\pi n_{i j}}{2 N^{2}}\right)
$$


which are constant and independent of $\vec{C}$. The latter form the gauge invariant moduli of these solutions, both on a lattice and in the continuum. If the action is non-zero, i.e. the twist is non-trivial, two of the moduli can also be identified with translational invariance, whereas translation in the other independent direction (which forms a zero eigenvector of the twist matrix) leaves the configuration invariant (on the lattice there is of course no continuous translation invariance). The stabilizer of this configuration is the subgroup of constant abelian gauge transformations, which is one dimensional. On the lattice this means that the Hessian has $3 N^{3}+2$ exact zero-modes and that $d T$ has the same number of unit eigenvalues (which we verified numerically). In the continuum we can even compute the Hessian exactly [11]. The eigen modes are products of a theta function (compare the study in the context of the "Copenhagen vacuum" [13]) and a plane wave (purely plane waves for abelian fluctuations). One easily shows that the gauge field is (anti) periodic in the $\vec{m}$ direction, i.e. $\vec{A}(\vec{x}+\vec{m} / e)=\tau_{3}^{\Delta} \vec{A}(\vec{x}) \tau_{3}^{-\Delta}$, with $e \equiv \mid$ g.c.d. $\left(m_{i}\right) \mid$ and $\Delta(\vec{m})=0$ or 1 , its value being determined from (repeated use of) eq. (33). The spectrum is now given by

$$
\lambda_{ \pm}(n, \ell)=2 \pi\|\vec{m}\|(2 n+1 \pm 2)+e^{2}((2 \ell+\Delta) \pi+\vec{C} \cdot \vec{m} / e)^{2} /\|\vec{m}\|^{2}, \quad \lambda_{o}(\vec{k})=(2 \pi \vec{k})^{2},
$$

The multiplicities are $2|e|$ for $\lambda_{ \pm}$and 2 for $\lambda_{o}$. The parameters $(n, \ell, \vec{k})$ run over $\left(\mathbb{N}, \mathbb{Z}, \mathbb{Z}^{3}\right)$. On the lattice $\lambda_{o}(\vec{k})$ will be replaced by $4 N^{2} \sum_{i} \sin ^{2}\left(\pi k_{i} / N\right)$, but the effect of the lattice on $\lambda_{ \pm}$is not expected to admit an analytic expression. In fig. 1a-c we plot the first few eigenvalues of the Hessian for resp. $\vec{m}=(0,0,1), \vec{m}=(0,0,2)$ and $\vec{m}=(1,1,1)$, computed numerically for $N=4$ and 6 in comparison with the exact continuum result as a function of $\vec{C}$, of which only the component along $\vec{m}$ is relevant. Only for $\vec{m}=(1,1,1)$ is $\Delta$ non-trivial.

A new feature that we believe not to have been observed so far, is that there are constant $(\neq 0)$ curvature solutions on a torus that are marginally stable. To be precise, for $\|\vec{m}\|=1$ and $|\vec{C} \cdot \vec{m}-\pi| \leq \pi-\sqrt{2 \pi}$ (using the $2 \pi$ periodicity of the spectrum in $\vec{C} \cdot \vec{m}$ we can restrict to $\vec{C} \cdot \vec{m} \in[0,2 \pi])$ there are no negative eigenvalues for the Hessian. We call this marginal stability, as one can first bring $\vec{C} \cdot \vec{m}$ outside the above range without changing the energy, and then let the configuration decay along one of the two unstable modes. For $\|\vec{m}\| \neq 1$ one can easily see that this effect doesn't occur.

Nevertheless, in all cases considered in fig. 1 the Hessian can have accidental zero eigenvalues for particular values of $\vec{C}$, such that potentially the algorithm can slow down dramatically. In table I we compare the gap $\hat{\mu}$ (the distance of the largest non-unit eigenvalue of $d T$ to 1$)$ with the predicted value $\hat{\mu}=\lambda_{m}^{2} /\left(14 N^{4}\right)$, determined from the smallest non-zero (absolute) eigenvalue $\lambda_{m}$ of the Hessian for $S$ (see eq.(31). The agreement is (perhaps surprisingly) very good. For $\vec{m}=(1,1,1)$ and $N=4$ there is considerable slowing down. What is called slowing down is of course dependent on the available computer power. We consider something slowed down considerably if (several) 10.000 or more sweeps are required to half the value of $\hat{S}$. In the case of slowing down, we could generally achieve good results by performing a small number of $S$ coolings, when the $\hat{S}$ cooling is being "stuck", sometimes after a (very) small random update on the links. That $S$ cooling can sometimes help can be argued for the case that $\lambda_{m}>0$ as follows. We have seen that to some degree the $\lambda_{m}$ eigenvector is also an eigenvector of $d T$, corresponding to the eigenvalue $\lambda=1-\hat{\mu}$. This should be the dominant mode along which the configuration differs from the true fixed point. All other modes, in particular the unstable ones, should have decreased significantly 
under the $\hat{S}$ cooling. If we don't cool too long with $S$, the unstable mode does not get a chance to grow, whereas the direction of the stable mode keeps on contracting (faster by a factor $\left.14 N^{2} / \lambda_{m}\right)$. With $\hat{S}$ cooling one can re-contract the unstable mode, which might have been grown during the $S$ cooling. We expect that the extra computational effort will not exceed by much what one would have to spend in trying to accelerate the algorithm, e.g. using fast-fourier [14, 7] or multi-grid [15] methods.

\begin{tabular}{|l|c|c|c|c|c|r|c|c|}
\hline$N$ & $\vec{m}$ & $\vec{C} \cdot \vec{m}$ & $\hat{S}$ & $S$ & $S_{\mathrm{th}}$ & $\lambda_{m}$ & $\hat{\mu}$ & $\hat{\mu}_{\mathrm{th}}$ \\
\hline 4 & $(1,1,1)$ & 3.472 & $2.90 \cdot 10^{-4}$ & 59.02774641 & 59.02761860 & 0.051 & $6.49 \cdot 10^{-7}$ & $7.31 \cdot 10^{-7}$ \\
6 & & 2.517 & $1.60 \cdot 10^{-2}$ & 59.17251733 & 59.18005528 & -0.574 & $1.74 \cdot 10^{-5}$ & $1.82 \cdot 10^{-5}$ \\
8 & & 3.175 & $2.65 \cdot 10^{-6}$ & 59.20573728 & 59.20573660 & 1.906 & & $6.34 \cdot 10^{-5}$ \\
\hline 6 & $(0,0,2)$ & 4.229 & $5.24 \cdot 10^{-7}$ & 78.75660845 & 78.75660838 & 4.084 & $8.58 \cdot 10^{-4}$ & $9.19 \cdot 10^{-4}$ \\
\hline
\end{tabular}

Table I: Results for the constant curvature solution on a $N^{3}$ lattice with magnetic flux $\vec{m}$. $S_{\text {th }}$ is the value obtained from the exact lattice result given in eq. (36), whereas $\hat{\mu}_{\mathrm{th}}=\lambda_{m}^{2} /\left(14 N^{4}\right)$, the predicted value for the distance of the largest non-unit eigenvalue of $d T$ to unity. The smallest (in absolute value) non-zero eigenvalue for the Hessian of $S$ is indicated by $\lambda_{m}$. This, as well as $\hat{\mu}$, was obtained from the exact lattice solution with the tabulated values of $\vec{C} \cdot \vec{m}$ extracted from the data.

Not only $\hat{S}$ itself is a good indication for how far one is from the fixed point. Also $d T$ can, and usually does, have an eigenvalue bigger than 1 if one is not close enough to the fixed point (the gauge modes always correspond to unit eigenvalues), whereas only at a fixed point will the gauge modes be exact zero-modes for the Hessian. All this provides us with more than enough criteria to be sure that we have an accurate lattice solution of the equations of motion. In table I we also compare the energy of the constant curvature solutions with the exact lattice result of eq. (36). The agreement is excellent, except perhaps for $N=6$ and $m=(1,1,1)$, but in that case we did not try to cool the configuration down to a very small value of $\hat{S}$. Nevertheless, the error in the energy is smaller than one part in 6000. Also quantities like the Polyakov loops agree very well with the exact lattice expressions, eq. (35).

\section{Results: The sphaleron}

We now discuss the results for the new sphaleron solutions. Some of the results are collected in table II. Also here we see that convergence of the $\hat{S}$ cooling algorithm can be well predicted in terms of $\lambda_{m}$. In figs. 2a-c we show scatter plots for the (complex) eigenvalues of $d T$ for the $\vec{m}=\overrightarrow{0}$ sphaleron, using a checkerboard updating for fig. 2a (to allow for vectorization of the algorithm) and a sequential updating for fig. $2 \mathrm{~b}$, both at $N=4$. We see that the checkerboard updating makes the bulk of the modes contract much faster (in addition to the gain of computational speed due to the vectorization). This remains true 
on larger lattices. In fig. $2 \mathrm{c}$ we show for comparison the checkerboard result for $N=8$. The eigenvalues that are responsible for the slowing down of the algorithm near the fixed point, in particular the eigenvalue closest to 1 , will only very weakly depend on the order in which one updates the links. Table II shows that the sphaleron with periodic boundary conditions $(\vec{m}=\overrightarrow{0})$ where $\lambda_{m} \sim 1.3$, has considerable slower cooling than the sphaleron with twisted boundary conditions, for which $\lambda_{m} \sim-10.8$. Nevertheless, we managed to obtain rather accurate results. In table II we also list $S, \hat{S}$ and the blocked Wilson action $S_{2 \times 2}$, for which the lattice spacing is effectively twice as large. Like the instantons of maximal size studied

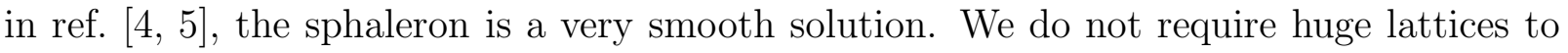
approach the continuum limit. Together with easily obtainable expansions in the lattice spacing $a=1 / N$, we have a large enough window (available values for $N$ ) to get accurate results. We can extract, as for the instantons (see ref. [4] for details), the continuum action by fitting to the formula

$$
S_{n \times n}=S_{0}\left(1-\alpha n^{2} / N^{2}-\left(\beta n^{4}+\gamma n^{2}+\delta\right) / N^{4}\right)+\mathcal{O}\left(n^{6} / N^{6}\right)
$$

We find for the twisted continuum sphaleron $(\vec{m}=(1,1,1))$ an energy $\mathcal{E}=34.148(2)$ and for the periodic one $(\vec{m}=\overrightarrow{0})$ we find $\mathcal{E}=72.605(2)$, with conservative error estimates.

\begin{tabular}{|r|c|c|c|c|c|c|c|}
\hline$N$ & $\vec{m}$ & $\lambda_{m}$ & $\hat{\mu}$ & $\hat{\mu}_{\mathrm{th}}$ & $S$ & $S_{2 \times 2}$ & $\hat{S}$ \\
\hline 4 & $(1,1,1)$ & -11.128 & $2.73 \cdot 10^{-2}$ & $3.46 \cdot 10^{-2}$ & 33.24878 & 29.77153 & $7.90 \cdot 10^{-6}$ \\
6 & & -10.900 & $8.74 \cdot 10^{-3}$ & $6.55 \cdot 10^{-3}$ & 33.77789 & 32.49357 & $2.28 \cdot 10^{-7}$ \\
8 & & -10.817 & & $2.04 \cdot 10^{-3}$ & 33.94548 & 33.28093 & $1.20 \cdot 10^{-11}$ \\
12 & & & & $4.03 \cdot 10^{-4}$ & 34.05973 & 33.78353 & $2.34 \cdot 10^{-6}$ \\
\hline 4 & $(0,0,0)$ & 1.704 & $7.33 \cdot 10^{-4}$ & $8.10 \cdot 10^{-4}$ & 68.13887 & 52.59651 & $2.73 \cdot 10^{-5}$ \\
6 & & 1.415 & $1.08 \cdot 10^{-4}$ & $1.10 \cdot 10^{-4}$ & 70.70174 & 64.36296 & $2.11 \cdot 10^{-5}$ \\
8 & & 1.301 & $2.41 \cdot 10^{-5}$ & $2.95 \cdot 10^{-5}$ & 71.55060 & 68.16790 & $6.93 \cdot 10^{-9}$ \\
12 & & & & $5.83 \cdot 10^{-6}$ & 72.14095 & 70.70631 & $4.13 \cdot 10^{-5}$ \\
\hline
\end{tabular}

Table II: Results for the sphalerons on a $N^{3}$ lattice with twisted and periodic boundary conditions. The smallest (in absolute value) non-zero eigenvalue for the Hessian of $S$ is indicated by $\lambda_{m}$. The predicted value for the distance of the largest non-unit eigenvalue of $d T$ to unity is given by $\hat{\mu}_{\mathrm{th}}=\lambda_{m}^{2} /\left(14 N^{4}\right)$. We also give the value of the Wilson action, its blocked value $S_{2 \times 2}$ and $\hat{S}$.

The evidence that these configurations are sphalerons follows from a study of the eigenvalues for the Hessian of $S$, whose low-lying values are listed in table III for $N=4,6$ and 8. We have verified that there are exactly $3 N^{3}+3$ zero-modes for both sphalerons, corresponding to the gauge modes and the three translational moduli (for $N>6$ the latter are indistinguishable from the gauge zero-modes, for $N=4$ their eigenvalues are not bigger than 0.01). Most importantly we have verified that there is precisely one unstable mode. Furthermore, we have not been able to find any other saddle point with a lower (non-zero) 
energy than the energies of the present configurations. For all practical purposes we thus consider the newly found solutions to be sphalerons.

We now wish to show that like on $S^{3}$ [3], these sphalerons are at the top of a tunnelling path. For the case of twisted boundary conditions this is the easiest to demonstrate, since the instanton with a continuum action of $4 \pi^{2}$, which was extensively studied on the lattice in ref. [6], has only the trivial translational and some discrete moduli. This means we only have to demonstrate that the top of the instanton path is identical to the sphaleron. There are two reasons why the top of an instanton path might not correspond exactly to a sphaleron. First, the lattice has a finite resolution and second, the extent in the time direction is finite. In particular the finite resolution causes problems because one needs to extrapolate along the tunnelling path the value of the energy and of $\hat{S}$. In particular the latter has a sharp dip near the top. A very large value of $N$ would be required to obtain a sufficient resolution. The effect of the finite extent in time is expected to be small since the instanton path approaches the vacua exponentially in time [6]. In fig. 3 we plot $\tilde{S} \sim \hat{S} / 64$ for each time-slice along the tunnelling path, cmp. ref. [5].

\begin{tabular}{|c|c|c|c|c|c|c|}
\hline \multicolumn{3}{|c|}{$\vec{m}=(1,1,1)$ sphaleron } & \multicolumn{3}{|c|}{$\vec{m}=\overrightarrow{0}$ sphaleron } & index 2 \\
\hline$N=4$ & $N=6$ & $N=8$ & $N=4$ & $N=6$ & $N=8$ & $N=8$ \\
\hline-11.1280 & -10.9003 & -10.8174 & -13.1960 & -13.3935 & -13.4432 & -13.1170 \\
\hline 0.0074 & 0.0001 & 0.0000 & 0.0073 & 0.0006 & 0.0000 & -0.7466 \\
\hline 0.0091 & 0.0001 & 0.0000 & 0.0124 & 0.0010 & 0.0000 & 0.0000 \\
\hline 0.0101 & 0.0001 & 0.0000 & 0.0139 & 0.0011 & 0.0000 & 0.0000 \\
\hline 13.1667 & 13.3371 & 13.3934 & 1.7041 & 1.4151 & 1.3009 & 0.0000 \\
\hline 13.1667 & 13.3371 & 13.3934 & 1.7070 & 1.4303 & 1.3012 & 3.2618 \\
\hline 13.1667 & 13.3372 & 13.3934 & 7.9677 & 8.0984 & 8.1260 & 5.8733 \\
\hline 16.0475 & 16.8663 & 17.1597 & 7.9682 & 8.1008 & 8.1261 & 5.8807 \\
\hline 16.0478 & 16.8663 & 17.1597 & 7.9684 & 8.1279 & 8.1266 & 6.1738 \\
\hline 16.0484 & 16.8663 & 17.1597 & 8.9984 & 10.2977 & 10.6604 & 8.5986 \\
\hline 21.4686 & 22.9444 & 23.4481 & 9.0189 & 10.3306 & 10.6611 & 8.6069 \\
\hline 21.4747 & 22.9444 & 23.4481 & 9.9919 & 10.8287 & 11.0701 & 15.0281 \\
\hline 21.8119 & 23.6594 & 24.2198 & 10.0256 & 10.8312 & 11.0701 & 16.7321 \\
\hline 21.8260 & 23.6594 & 24.2198 & 10.0354 & 10.8594 & 11.0707 & 19.7489 \\
\hline 25.0998 & 26.1760 & 26.4962 & 24.6675 & 26.4047 & 26.9618 & 20.6039 \\
\hline 25.1050 & 26.1760 & 26.4962 & 24.6703 & 26.4205 & 26.9621 & 21.3605 \\
\hline
\end{tabular}

Table III: The lowest lying eigenvalues for the Hessian of $S$ for the twisted and periodic sphaleron on a $N^{3}$ lattice for $N=4,6$ and 8. Also is given the spectrum for the periodic solution with two unstable modes (index 2), close in energy to the sphaleron, discussed in the text (see also table IV). 
We take the configuration at the top of the instanton path, where $\tilde{S}$ is minimal, and cool it with $\hat{S}$. This way we easily obtain the sphaleron solution. As $\hat{S}$ decreases monotonically under the $\hat{S}$ cooling, and as $\hat{S}$ is already small at the top of the instanton path, we expect that the instanton path goes through a sphaleron. Further evidence is provided by calculating the quantity

$$
D_{N}(t)=4\left\|U_{t}-U_{s}\right\|^{2} / N \sim \int d_{3} x\left(\delta A_{i}^{a}(\vec{x})\right)^{2}
$$

where the latter expression is obtained in the continuum limit. $U_{t}$ is the configuration at the time $t$ along the instanton path and $U_{s}$ is the sphaleron configuration, obtained after cooling from the configuration $U_{t}$ (for this, $t$ should be chosen not too far away from the top of the barrier, as otherwise cooling will bring the configuration to the vacuum). As the cooling algorithm commutes with gauge transformations, this gives a gauge invariant measure for the distance of the two configurations. Indeed, computing $\left\|U_{t}-U_{t^{\prime}}\right\|$ gives huge values, as the gauge between different time slices will differ randomly. For the configurations of fig. 3 we find $D_{8}(0)=0.0246, D_{8}(1 / 8)=1.162, D_{8}(-1 / 8)=1.5713$ and $D_{12}(0)=0.0340$. We have indicated also the values at either side of the top of the barrier for $N=8$ to show that, not being exactly at the top can have a sizable effect on $D_{N}(0)$ (and on $\tilde{S}(t=0)$ ). We believe the numerical evidence is sufficiently convincing to conclude that the instanton path does go through a sphaleron. In fig. 4 we give for $N=12$ the two dimensional cross sections of $\mathcal{E}_{B}(x, y, z)$ at the twelve lattice values of $x$. Because of the cubic symmetry of the sphaleron, the other cross-sections have identical profiles (modulo translations).

For the case of periodic boundary conditions the instantons have additional moduli. We did provide evidence in ref. [4 that for $T \rightarrow \infty$ the moduli are described by the scale, translational and vacuum parameters. The latter being specified by the Polyakov loops $P_{i}(t=\infty)=-P_{i}(t=-\infty)$ (we have not yet proven or disproven if this relation at $t= \pm \infty$ can be relaxed). In particular we probed the dependence of the tunnelling path on $P_{3} \equiv P_{3}(t=-\infty)$, keeping the other two equal to unity. In practise we can of course not reach $|t|=\infty$, and this is a problem for $P_{3}= \pm 1$. In that case one can show that the approach to the vacuum for large $t$ is no longer exponential due to the quartic behaviour of the potential in the zero-momentum sector. We demonstrate the sensitivity of the instanton path on the extent in the time direction for $N=8$ and $N_{t}=24,48$ in fig. 5 . We see that for large $N_{t}$ the tails in the electric energy [4, 5] are lowered considerably, while the magnetic energy at the top of the barrier (not visible at the scale of fig. 5) is lowered from $S(\varepsilon=-1)=74.041$ at $N_{t}=24$ to 73.465 at $N_{t}=48$, actually below the value of 73.923 obtained for $P_{3}=0.5$ in ref. [5] (for which we did not observe such a sensitivity in $N_{t}$ ). It means that there is near degeneracy in energy at the top of the barrier as a function of $P_{3}$. The variation of the energy as a function of $P_{3}$ can be so small, because the energy functional near the sphaleron has two rather shallow directions (associated to the two eigen modes with $\lambda_{m} \sim 1.3$ ). To investigate if our sphaleron indeed corresponds to the top of a tunnelling path, presumably the one with $P_{3}=1$, we took the configuration at the top of the energy barrier for the values of $P_{3}$ that were considered in ref. [5] and applied $\hat{S}$ cooling for 8000 sweeps on each, such that the energy and $\hat{S}$ stabilized, and further cooling would make the configuration only move (very slowly) along the slowest mode in the cooling algorithm. We discovered a very useful set of parameters to distinguish the various configurations thus obtained. It turns out that the energy profile is almost identical 
for each configuration, that the profiles for the Polyakov lines only vary weakly, but that the vector

$$
\vec{b} \equiv\left(\operatorname{Tr}\left(B_{1}^{2}\right), \operatorname{Tr}\left(B_{2}^{2}\right), \operatorname{Tr}\left(B_{3}^{2}\right)\right) / \sqrt{\frac{1}{3} \sum_{i} \operatorname{Tr}\left(B_{i}^{2}\right)^{2}}
$$

averaged over the lattice $(<\vec{b}\rangle)$, clearly differentiates the various configurations. The sphaleron solution distinguishes itself by a symmetric value $\vec{b}=(1,1,1)$ at each point independently. In all other cases the variance of the parameter $\vec{b}$ is non-zero. To a good degree $<b_{1}>=<b_{2}>$, which is of course a direct consequence of the symmetry of the lattice and of the fact that $P_{1}=P_{2}$. We also compute $D_{8}^{\prime}$, which is defined as in eq.(39) but with $U_{t}$ the configuration obtained after the $\hat{S}$ cooling. For this it is important to note that the instantons in table IV were all generated from the same random start at $P_{3}=1$ (or $C_{3}=0$; see ref. [5]). This means that each is in the same gauge and $D_{8}^{\prime}$ is a good gauge invariant measure for the distance of the various cooled configurations to the sphaleron. In table IV we collect the results thus obtained, and see that after 8000 cooling sweeps $\hat{S}$ is dramatically lowered also for $P_{3}=0$. If we compute the Hessian for this configuration we find a Morse index of two, i.e. there are two unstable modes (see table III for the low-lying eigenvalues). Note that $S$ for this configuration is only slightly higher than for the sphaleron, and that $\lambda_{m}=-0.74655$ will cause an even slower cooling than for the sphaleron. We have performed 8000 extra $\hat{S}$ cooling sweeps before computing the Hessian, but the quality of its zero modes were sufficiently good to trust the cooled configuration for $P_{3}=0$ to be close enough to a solution. We notice that except near $P_{3}=1$, the value of $\langle\vec{b}\rangle$ at the top of the barrier (denoted by $\left\langle\vec{b}_{t}\right\rangle$ in table IV) is changed after 8000 $\hat{S}$ cooling sweeps. We conclude that the index 2 solution is not at the top of any of the instanton paths we considered here.

\begin{tabular}{|c|c|c|c|c|c|c|}
\hline$C_{3}$ & $<\vec{b}_{t}>$ & $<\vec{b}>$ & $S$ & $S_{2 \times 2}$ & $\hat{S}$ & $D_{8}^{\prime}$ \\
\hline 0 & $(1.01,1.01,0.99)$ & $(1.01,1.00,0.99)$ & 71.5507 & 68.1680 & $2.08 \cdot 10^{-4}$ & 0.000 \\
$\pi / 5$ & $(1.02,1.02,0.96)$ & $(1.03,1.02,0.95)$ & 71.5547 & 68.1732 & $9.77 \cdot 10^{-3}$ & 0.056 \\
$2 \pi / 5$ & $(1.05,1.05,0.89)$ & $(1.07,1.06,0.85)$ & 71.5913 & 68.2205 & $7.53 \cdot 10^{-2}$ & 0.100 \\
$2 \pi / 3$ & $(1.10,1.10,0.77)$ & $(1.14,1.13,0.65)$ & 71.7569 & 68.4322 & $1.58 \cdot 10^{-1}$ & 0.560 \\
$4 \pi / 5$ & $(1.11,1.11,0.73)$ & $(1.15,1.15,0.60)$ & 71.8301 & 68.5250 & $1.18 \cdot 10^{-1}$ & 0.894 \\
$\pi$ & $(1.12,1.12,0.69)$ & $(1.17,1.16,0.53)$ & 71.9318 & 68.6537 & $2.60 \cdot 10^{-4}$ & 2.186 \\
\hline
\end{tabular}

Table IV: Results for $N=8$ associated to the top of the instanton path (obtained with $S(\varepsilon=-1)$ cooling, see ref. [5]) that interpolates from the vacuum specified by $P_{1}=P_{2}=1$ and $P_{3}=\cos \left(C_{3} / 2\right)$ at $t=-\infty$, to the vacuum specified by $P_{1}=P_{2}=-1$ and $P_{3}=-\cos \left(C_{3} / 2\right)$ at $t=\infty$. The vector $\vec{b}$ is the parameter defined in eq. (40). From the third column on are tabulated the results after $8000 \hat{S}$ cooling sweeps. $D_{8}^{\prime}$ is defined as in eq. (39), with $U_{t}$ the cooled configuration. Both from the first and last case one finds solutions of the equations of motion, resp. the sphaleron and the index 2 saddle point. See table III for their Hessians. 
Finally, we calculated $D_{N}(t)$ from the configuration in table IV with $C_{3}=0\left(P_{3}=1\right)$ after further $S(\varepsilon=-1)$ cooling on the instanton, as represented in fig. 5 (at $\left.N_{t}=24\right)$. We find $D_{8}(0)=0.0496, D_{8}(-1 / 8)=2.212, D_{8}(1 / 8)=3.369$ and $D_{12}(0)=0.0368$ (not represented in fig. 5). Taking into account the time-tail effects discussed before and the fact that the instanton path was generated with $S(\varepsilon=-1)$ cooling, whereas $\hat{S}$ cooling was performed at $\varepsilon=1$, the quality of these results are sufficient to conclude that the sphaleron lies on the instanton path that has $P_{i}(t=-\infty)=-P_{i}(t=\infty)= \pm 1$. In fig. 6 we give the results for the energy distribution on a $12^{3}$ lattice.

\section{Conclusions}

The main motivation for this paper was to find the sphaleron solution for pure $\mathrm{SU}(2)$ gauge theories in a finite volume and to show that it is at the top of the barrier that separates two classical minima, connected by an instanton solution with minimal action. We studied both the case of twisted and periodic boundary conditions. For the latter the situation was rather subtle because of the many additional moduli in the instanton parameter space. Furthermore, at the sphaleron there are two very flat directions for the energy functional, and we found a saddle point with two unstable modes not more than $\delta \mathcal{E}=0.5$ above the sphaleron. The sphaleron solution for the periodic case has some very special properties. Like for the twisted case [6] only low fourier components seem to dominate, but especially the property that locally $\operatorname{Tr}\left(B_{i}\right)^{2}$ is independent of $i$ is intriguing. There is good hope that in the future this problem might be tractable analytically. In any case it presents an interesting challenge.

To achieve all these results we built on the idea that one can find unstable solutions to the equations of motion by minimizing the functional obtained by squaring the gradient of the field equations [7, 9]. What we have added is a specific algorithm for $\mathrm{SU}(2)$ to minimize this functional in a deterministic way. This allowed us to analyse in detail the convergence of the algorithm, also providing results for the standard cooling algorithm [10. It is likely that the algorithm can be extended to include a scalar sector so that we can also study the sphaleron in the standard model [1]. In any case one can always try to minimize the functional by doing random updates. One can easily think of many more applications worthwhile pursuing with these methods.

\section{Acknowledgements}

We have benefited from discussions with Jim Hetrick, Jan Smit, Arjan van der Sijs and Jeroen Vink. The eigenvalues of the linearized algorithm and Hessian were determined using the EISPACK [16] routines. We thank Willem Vermin for providing the source codes. Figs. 4 and 6 were produced using Mathematica [17], its programs with the raw data for the energies are attached to the electronic hep-lat bulletin board version of this paper. This work was supported in part by grants from "Stichting voor Fundamenteel Onderzoek der Materie (FOM)" and "Stichting Nationale Computer Faciliteiten (NCF)" for use of the CRAY Y-MP and C98/4256 at SARA. M.G.P. was supported by a Human Capital and Mobility EC fellowship. 


\section{References}

[1] F. R. Klinkhamer and M. Manton, Phys. Rev. D30 (1984) 2212.

[2] C. Taubes, Comm. Math. Phys. $\underline{86}$ (1982) 257, 299;

C. Taubes, in: Progress in gauge filed theory, eds. G. 't Hooft et. al., Plenum Press, New York, 1984, p.563.

[3] P. van Baal and N. D. Hari Dass, Nucl. Phys. B385 (1992) 185.

[4] M. García Pérez, A. González-Arroyo, J. Snippe and P. van Baal, Nucl. Phys. B413 (1994) 535.

[5] M. García Pérez, A. González-Arroyo, J. Snippe and P. van Baal, On the top of the energy barrier, Leiden preprint INLO-PUB-14/93, to appear in the proceedings of Lattice '93, Nucl.Phys. B(Proc.Suppl.) (1994).

[6] M. García Pérez, A. González-Arroyo and B. Söderberg, Phys. Lett. B235 (1990) 117;

M. García Pérez and A. González-Arroyo, J. Phys. A26 (1993) 2667.

[7] A. Duncan and R.D. Mawhinney, Nucl. Phys. B(Proc.Suppl.)26 (1992) 444; Phys. Lett. B282 (1992) 423.

[8] K. Wilson, Phys. Rev. D10 (1974) 2445.

[9] A.J. van der Sijs, Nucl. Phys. B(Proc.Suppl.)30 (1993) 893; Phys. Lett. B294 (1992) 391.

[10] B. Berg, Phys. Lett. 104B (1981) 475;

J. Hoek, M. Teper and J. Waterhouse, Nucl. Phys. B288 (1987) 589.

[11] P. van Baal, Comm. Math. Phys. $\underline{94}$ (1984) 397.

[12] G. 't Hooft, Nucl. Phys. B153 (1979) 141.

[13] J. Ambjørn and P. Olesen, Nucl. Phys. B170[FS1] (1980) 60;

J. Ambjørn, B. Felsager and P. Olesen, Nucl. Phys. B175 (1980) 349.

[14] C.T.H. Davies, et al, Phys. Rev. D37 (1988) 1581.

[15] A. Hulsebos, M.L. Laursen and J. Smit, Phys. Lett. B291 (1992) 431.

[16] B.T. Smith, et al, Matrix Eigensystem Routines - EISPACK Guide, second edition (Springer, New York, 1976); B.S. Garbow, et al, Matrix Eigensystem Routines - EISPACK Guide Extension (Springer, New York, 1977).

[17] S. Wolfram, et. al., Mathematica (Addison-Wesley, New York, 1991). 


\section{Figure captions}

Figure 1: The low-lying eigenvalues of the Hessians for the constant curvature solutions specified by (a) $\vec{m}=(0,0,1)$, (b) $\vec{m}=(0,0,2)$ and (c) $\vec{m}=(1,1,1)$ at $N=4$ (dotted lines), $N=6$ (dashed lines) and $N=\infty$ (i.e. the continuum). Each level is two-fold degenerate except for $\vec{m}=(0,0,2)$ and the levels indicated by the arrow in (a), which are all four-fold degenerate.

Figure 2: The scatter plots for the complex eigenvalues of $d T$ for the periodic sphaleron at $N=4(\mathrm{a}, \mathrm{b})$ and $N=8$ (c), for the latter we also show the blown-up region near $\lambda=1$. Figs. (a,c) were generated with a checkerboard updating, whereas fig. (b) was generated with sequential updating.

Figure 3: The value of $\tilde{S}(t) \sim \hat{S}(t) / 64$ (at $\varepsilon=1$ ) for the twisted instanton [6] with continuum action $4 \pi^{2}$ on a $8^{3} \times 24$ (triangles) and a $12^{3} \times 36$ (squares) lattice. The strong dip in $\tilde{S}(t)$ at $t=0$ indicates stationarity of the energy functional due to the tunneling through a sphaleron.

Figure 4: The profile for the magnetic energies $\mathcal{E}_{B}$ for the twisted sphaleron on a $12^{3}$ lattice at fixed values of $x$ as a function of $y$ and $z$. The lattice is represented by the grid-lines (all coordinates are interchangeable). The vertical axes runs from 0 to 110, in units of the inverse physical length of the box.

Figure 5: The electric and magnetic energies obtained after cooling with $S(\varepsilon=-1)$ for a periodic $8^{3}$ lattice in the space directions and for boundary conditions fixed in the time direction of $N_{t}$ sites to $P_{i}=-1$ at one end and $P_{i}=1$ at the other end, for $N_{t}=24$ (squares for $\mathcal{E}_{E}$ and crosses for $\mathcal{E}_{B}$ ) and $N_{t}=48$ (triangles for $\mathcal{E}_{E}$ and stars for $\mathcal{E}_{B}$ ). The time-tails are plotted at a blown-up scale to show the electric tails [t] that distort the instanton solution at small $N_{t}$.

Figure 6: The profile for the magnetic energies $\mathcal{E}_{B}$ for the periodic sphaleron on a $12^{3}$ lattice at fixed values of $x$ as a function of $y$ and $z$. The lattice is represented by the grid-lines (all coordinates are interchangeable). The vertical axes runs from 0 to 110, in units of the inverse physical length of the box. 
This figure "fig1-1.png" is available in "png" format from: http://arxiv.org/ps/hep-lat/9403026v1 

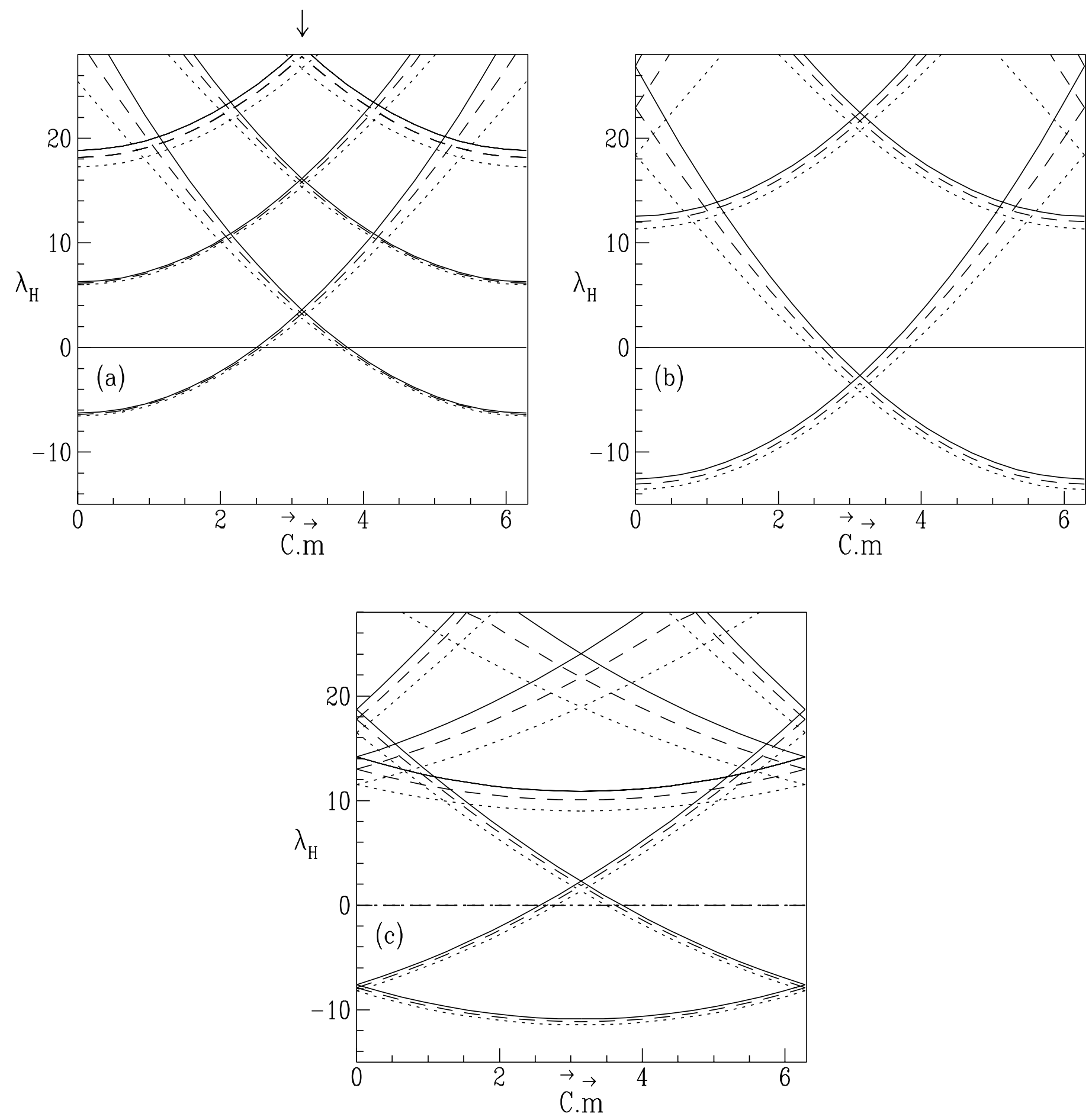
This figure "fig2-1.png" is available in "png" format from: http://arxiv.org/ps/hep-lat/9403026v1 
This figure "fig1-2.png" is available in "png" format from: http://arxiv.org/ps/hep-lat/9403026v1 
This figure "fig2-2.png" is available in "png" format from: http://arxiv.org/ps/hep-lat/9403026v1 

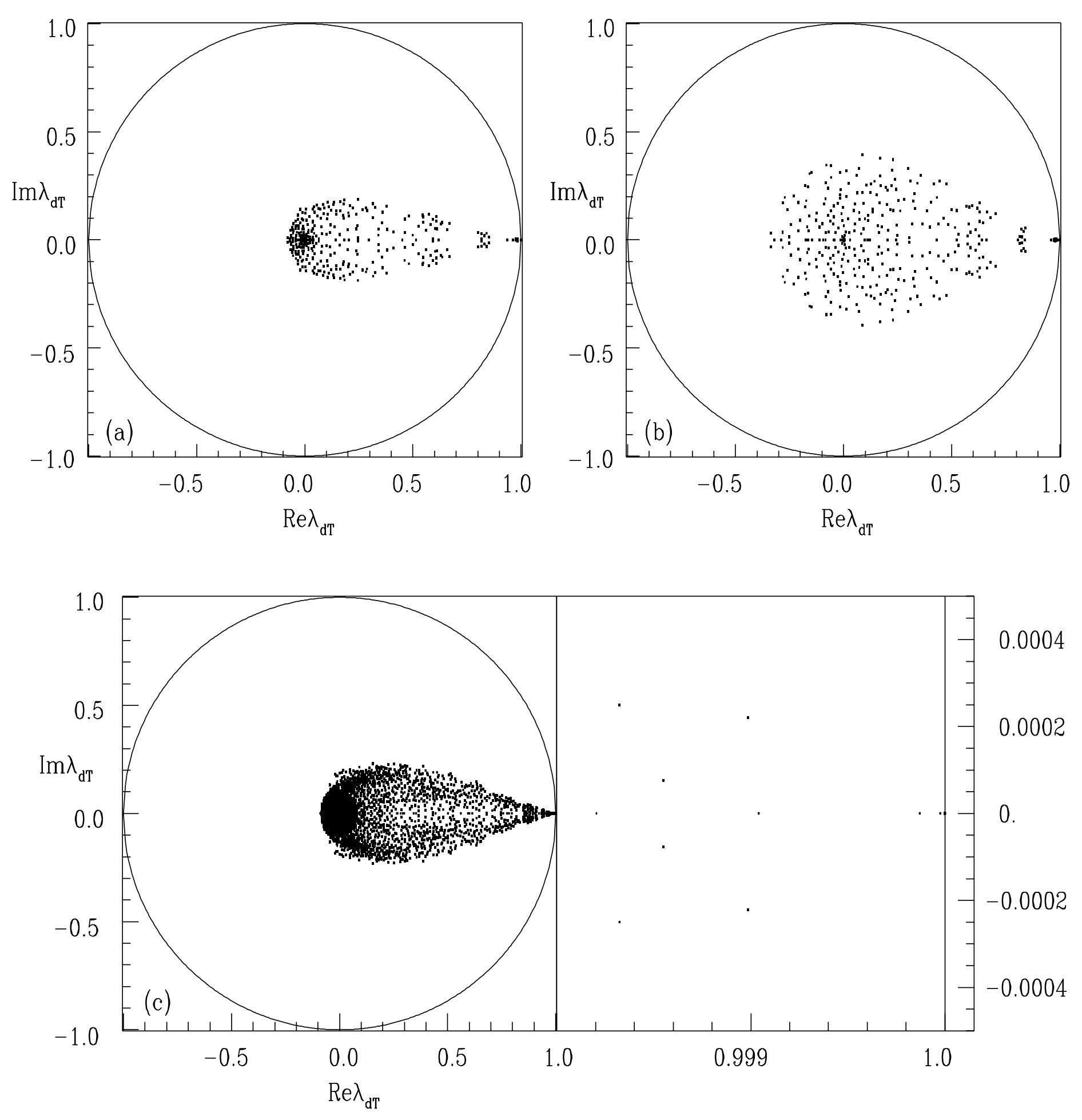
This figure "fig1-3.png" is available in "png" format from: http://arxiv.org/ps/hep-lat/9403026v1 


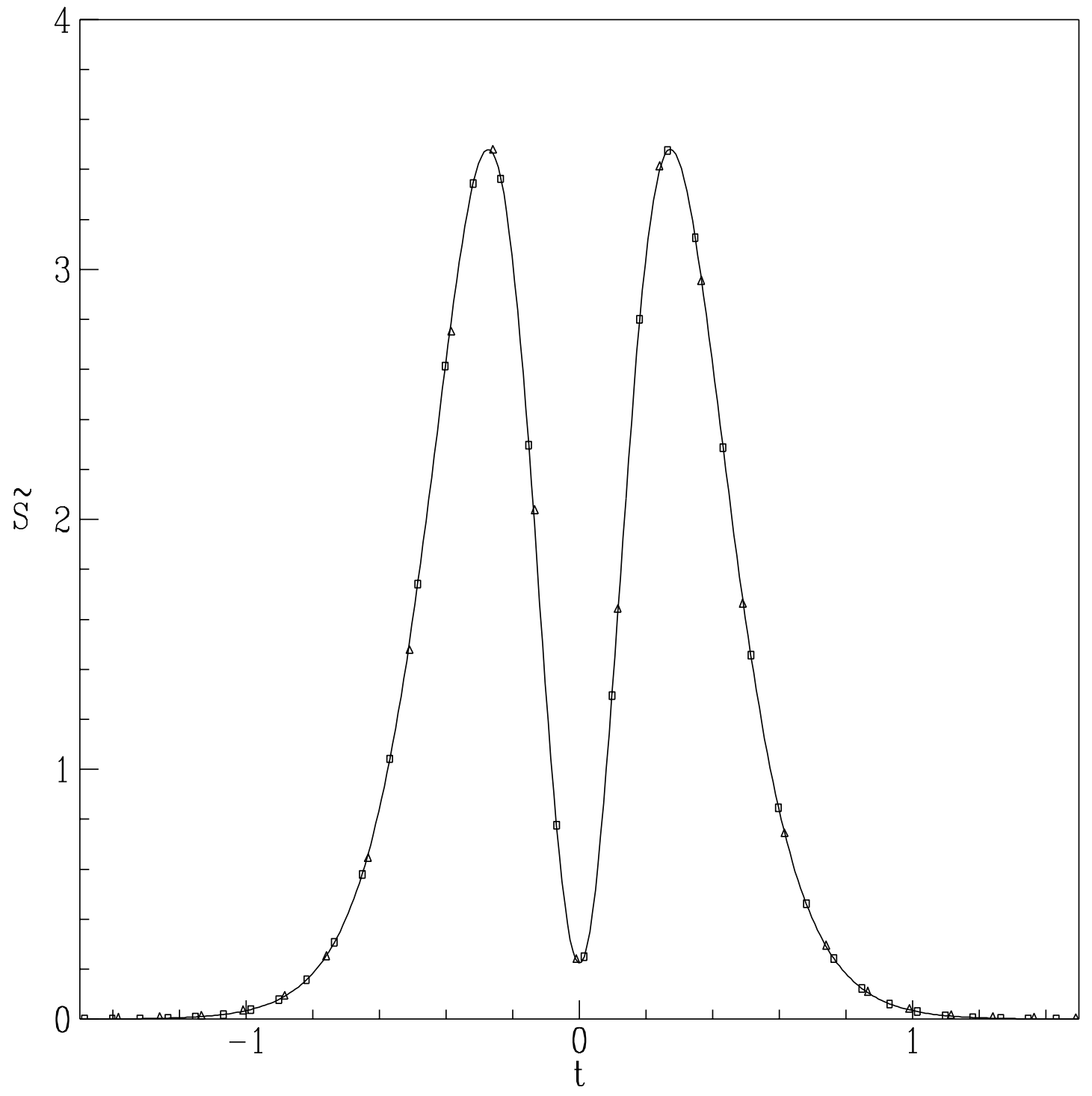


This figure "fig1-4.png" is available in "png" format from: http://arxiv.org/ps/hep-lat/9403026v1 


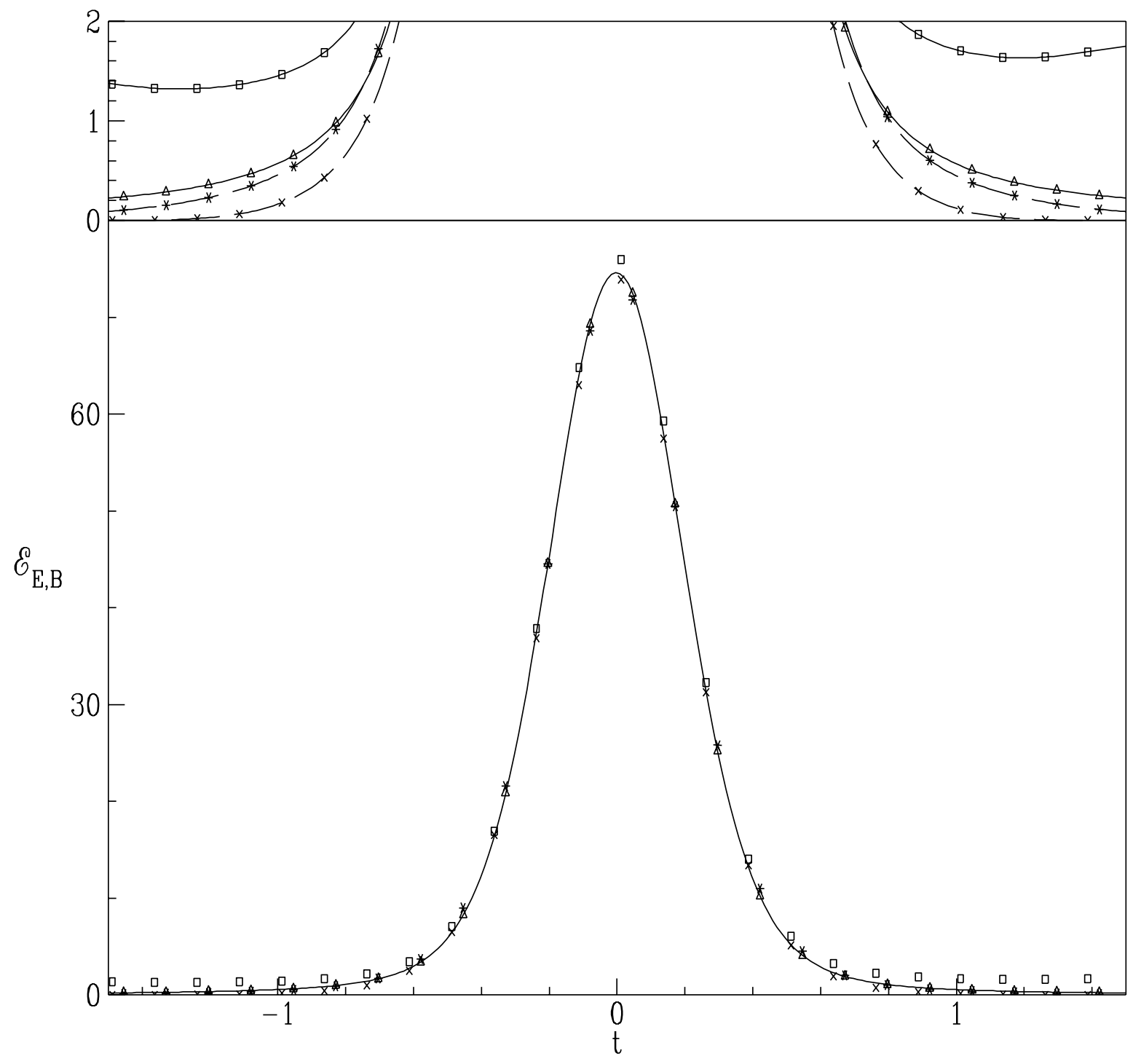

